\title{
DIABETES IN CHILDREN
}

\author{
By JAMES Robertson, M.B., M.R.C.P. \\ Consultant Physician to St. Andrew's and St. Clement's Hospitals, Bow, Brentford Hospital, Teddington Hospital and \\ Willesden General Hospital; \\ Consultant in Diabetes to Palingswick Hostel for Diabetic Children (L.C.C.)
}

The metabolic disorder of diabetes mellitus can occur at any time of life. Its occurrence is very rare under one year and rare under two years. After this age, its incidence tends to increase, and in children of ro to 15 years it is not uncommon. The exact incidence of diabetes in children in this country is not known.

In children, its onset is usually acute, with symptoms of loss of weight, polyuria, thirst and lassitude. It is unusual for symptoms to be present longer than three or four weeks before the diagnosis is made. Occasionally the onset may be very acute with diabetic coma occurring within a day or so of the first symptom. In such cases coma is preceded by vomiting.

Marked loss of weight is often an early symptom and is associated with unusual tiredness, lack of energy and loss of interest. As a paradox a voracious and insatiable appetite may accompany these symptoms, sometimes with a craving for sugar and sweets. The thirst of the child developing diabetes may be extreme and is almost indescribable. No matter how much liquid is drunk the thirst is still present and extreme. Some children complain that their tummies are so full of water that they cannot eat to ease their enormous appetite.

Except in very few cases ketosis is present in all new diabetic children, as shown by the Rothera's nitroprusside test. As the condition develops and becomes worse, increasing amounts of ketone bodies are found in the urine and the odour of acetone appears in the breath. When the Gerhart's ferric chloride test becomes positive, the diabetic condition is serious and the further progress to vomiting and diabetic coma is not far away. As in some adults, severe ketosis may cause abdominal pain, not necessarily with vomiting. The pain may be severe and the differential diagnosis from an acute surgical condition may be very difficult. It must be remembered that this condition can happen at any time in the child's diabetic life when for any reason diabetic control is lost and ketosis occurs.
Two further symptoms require brief mention. Bed-wetting and vulval irritation. Unusual bedwetting is often a presenting symptom of diabetes and stops when the diabetes is controlled. Bedwetting by the known diabetic usually means loss of control sometimes only to a slight degree. Vulval irritation occurs in girls as in adult women, due to the physical irritation of the sugar. It clears up as soon as the sugar improves.

The untreated diabetic child is susceptible to all intercurrent infections. Boils and other skin sepsis, pneumonia and pulmonary tuberculosis may occur. The new case presenting with a very sudden onset of symptoms leading rapidly to precoma or coma should be thoroughly searched for signns of some infection.

\section{Treatment}

The general principles of treatment of diabetic children varies little from that of adults. There can, however, be no discussion as to whether insulin should be given or not. All diabetic children must have insulin even in those very rare cases where symptoms are mild and apparent control can be achieved by dietary restrictions. Insulin is necessary for the child's proper growth and development and must be given. Tolbutamide or other oral preparations have as yet no place in the treatment of the diabetic child.

There have been in the past varying opinions on the method of controlling diabetes, and how necessary is the control of the blood sugar. Most authorities are now agreed that some measure of control of the blood sugar is necessary-indeed, vital-to the diabetic child. The method of allowing the child to eat what it likes and to give insulin to prevent symptoms and ketosis is fraught with future danger to the child. Constant glycosuria and a high blood sugar leads to ill health and the development of diabetic vascular complications in later life.

In a survey ${ }^{1}$ of .203 diabetic patients having diabetes more than 10 years and starting diabetes before the age of 15 years, it was shown that the 
vascular complications of diabetes occurred with greater frequency and earlier in poorly-controlled cases than in well-controlled cases. Good control was defined simply as when the midday blood sugar is below $200 \mathrm{mg}$. per cent., and the first morning specimen of urine contains only traces of or no sugar.

How to obtain good control of diabetes in children is always a difficult task. The principles are the same in children as in adults. There has to be a balance between the intake of carbohydrate foods and the insulin given. Protein foods, although contributing some available carbohydrate, do not in practice materially affect the level of the diabetic's blood sugar. No restriction to reasonable quantities of protein foods is made. Carbohydrate foods, on the other hand, must be controlled and taken in constant amounts at regular times. The amount of carbohydrate allowed any individual child does not really matter as long as the appetite is satisfied and as long as he or she eats the same amount of carbohydrate at each meal each day. Food is to most children one of the most important things in life, and if they do not get enough to eat they tend to find ways and means of getting it, thus upsetting the control of their diabetes and precipitating ketosis or diabetic coma. To avoid this, diets liberal in carbohydrate foods are allowed and enough insulin given to control the blood sugar. Some children may need as much as $300 \mathrm{~g}$. of carbohydrate a day, and the only condition laid on such large diets is that the child shall always eat it. So important is this constant intake of carbohydrate that it may be said that " the diet is the foundation stone on which the whole success of good diabetic control is laid.'

\section{Insulin}

The diabetes of children is acute and severe. At the onset it is usually insulin sensitive but the blood sugar tends to rise and fall over the day very markedly. Briefly, this type of diabetes is 'labile' and needs a quick-acting powerful insulin to control it. As time progresses the diabetes tends to become less insulin sensitive and larger doses are necessary to maintain control. This is particularly noticeable at or approaching puberty and with any intercurrent infections. After each severe cold or infectious fever the child's insulin dose is usually found to be higher than before. The infection itself causes severe disturbance of the diabetic balance, causes heavy glycosuria and often ketosis; sometimes even precipitating diabetic coma. The only insulin which can successfully surmount these difficulties is soluble insulin given twice a day. Furthermore, quick adjustments to insulin dosage can be made for heavy sugar found due to dietary indiscretions. The long-acting insulins, viz. protamine zinc, insulin zinc suspension (Lente), globin or isophane have little place in the treatment of diabetic children, but PZI and isophane or globin may be valuable to prolong the action of soluble insulin in certain cases.

The one advantage of these long-acting insulins - that of having to be given only once a daywould seem ideal for children, but the control given by this method is in the main not good enough to outweigh the technical advantage. Most children learn to give their own insulin remarkably quickly and are so adaptable that two injections a day is little disadvantage. It is only in the very young that one injection a day is of value and most of these cases are, and remain, sufficiently insulin sensitive for good control to be obtained on Lente insulin once a day. The parents must, however, be warned from the outset that the child will need two injections a day later so that there is no shock or surprise when the occasion arises. The change can usually easily and safely take place when the child is about seven or eight years old, and when he or she can give their own injections.

\section{Diet}

The distribution of the carbohydrate in the diet is an important factor in obtaining good control, as also is the adjustment of the insulin. The diet must be arranged so that the highest proportion of carbohydrate is eaten at the time of maximum action of the insulin. The feeding habits of the child must also be considered. The important meal, 'tea,' commonly taken at 4 or 4.30 p.m., should be substantial and, in children, regarded as a 'main' meal. This necessitates the insulin, where two injections of soluble insulin are used, to be given before tea, and in children this works out well in practice. The following is an approximate distribution of carbohydrate for a diet at 200 g. carbohydrate per day.

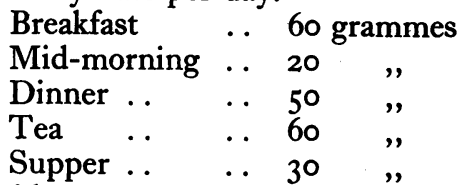

The mid-morning 'buffer' is an essential to prevent undue lowering of the blood sugar and consequent hypoglycaemia before dinner. The supper also carries the child through the night without hypoglycaemia. It may be found for individual children that changes have to be made. The mid-morning may have to be increased to $30 \mathrm{~g}$. and the supper likewise to $40 \mathrm{~g}$. For bigger or smaller amounts of carbohydrate in the diet the same proportions should be tried.

When 'balancing' the child the times of taking the urine tests are important. Urine tests for 
sugar and acetone should be done on specimens passed before each meal. It is pointless to test specimens passed after meals, particularly the main meals, because even in the well controlled, sugar will be passed after meals. Midday is a useful time to take the blood sugar as the result of this test will reflect how the morning insulin is working.

The estimation of the initial dose of insulin for any given child is a matter of experience. The size of the dose depends on the severity of the symptoms and degree of ketosis-the more severe the symptoms and heavier the ketosis, the larger the dose of insulin required.

Depending on these factors and the age of the child, the initial dose should be between 16 and 30 units of soluble insulin twice a day. The evening dose should be slightly lower than the morning dose given 15 to 20 minutes before breakfast and tea. Then according to the results of the previous day's tests the insulin adjusted up or down by 2 or 4 units.

It may be found in some cases that the morning urine test shows heavy sugar and some ketosis, and in these cases the child may be thirsty in the mornings. This shows that the evening dose of soluble insulin has not carried through the night and that the diabetes has relapsed. This is easily eorrected by adding a small dose of 4 to 8 units of protamine zinc insulin to the evening dose of soluble insulin.

When, in very young children, it is decided to give a single dose of a long-acting insulin, Lente insulin is probably the best. The initial dose should be between 12 to 24 units according to the symptoms and ketosis. The dietary carbohydrate should be divided fairly evenly throughout the day, giving a little less at breakfast. The insulin injection should be given half an hour before breakfast. The insulin should be varied according to the results of the usual tests and sometimes it may be necessary to alter the distribution of carbohydrate in the diet.

\section{The Injection}

Most children learn the technique of giving their own insulin injections easily and quickly. Some take time to learn and require great encouragement. For those who find great difficulty, it is sometimes helpful to advise an automatic injector, rather like a gun, which on pressing a trigger will insert the syringe needle painlessly.

The first injections of insulin are sometimes a little painful, but the tissues soon become used to it and the injections become painless. Occasionally, local sensitivity occurs in the form of red, itchy and sometimes painful bumps at the site of injection. These usually disappear in a week or so but, if severe, can be relieved by giving an antihistamine by mouth. When, very rarely, severe urticarial reactions occur, these, too, are relieved by anti-histamines.

It is important to impress on the child and parent the importance of changing the site of the injection and never giving the injection into the same situation over any period of time. Children are very apt to become fond of one place and to always give their injection there because the skin $\nRightarrow$ becomes insensitive and there is little or no pain. This causes an 'insulin tumour' and absorption from the site is slowed down and very variable. It is a common cause of marked variations in the child's diabetic control.

Some children may develop fat atrophy at or near the site of injections. It takes months to develop and has the appearance of unsightly hollows in the skin. The condition is harmless and improvement may be obtained by continuing to give the injections of insulin into the hollow area.

\section{Education}

The initial stabilization should take place in hospital. From the outset, the child and the $\overrightarrow{0}$ responsible parent must be taught all the factore of involved in keeping the diabetes controlled. The. parent should be encouraged to see the child as much as possible, be taught how to measure and give insulin injections. The dietary arrangement must be fully explained and food values and how to vary the diet taught. It is too much to expect the child to learn everything in the short time in hospital, so it will rest with the parent to gradually instruct the child and-particularly with younger children-teach how to measure and give their own insulin. The parent must also learn how to adjust the insulin dose and how to spot hypoglycaemia and how to deal with it. It is wise to deliberately give the child a hypoglycaemic attack so that the warning signs can be recognized and treated with sugar at once. Urine testing must be taught and emphasis laid on the best times to test. Once the child's diabetes is balanced, urine tests need be done less frequently. A test on a specimen passed immediately before dinner-the bladder having been emptied up to an hour before, will show if the morning insulin is sufficient and according to this test so it can be altered or left as it was. The bedtime and first morning specimens similarly show how the evening insulin is working. These tests should be done twice or three times a week.

Follow-up visits of the child with the parent should be made regularly every three or four weeks at first and, if all goes well, the time between visits widened up to three months. 


\section{Hypoglycaemia}

This condition in children is troublesome until the child has learnt to take the necessary precautions to avoid it as far as possible. Diabetes in children may vary, and insulin requirements vary for no very obvious reason. Insulin reactions may herald a phase of lessening insulin requirements and reactions for no good reason indicate that less insulin is required at the time of the dose causing reactions.

Exercise, the great variable in children's lives, will cause hypoglycaemia when taken in more than usual amounts. The child has to learn that before playing games, swimming or doing anything which will be more energetic than usual, extra carbohydrate must be eaten, either as bread or sweets. The amount to eat has to be learnt by trial and error and experience of the child and parent. As with exercise, so eating too little carbohydrate may cause an insulin reaction, as also will the inadvertent taking of too much insulin.

The onset of hypoglycaemia is usually recognized by the child, but sometimes, when preoccupied by playing or other matters, the early symptoms may be ignored and severe hypoglycaemic symptoms occur. The parent, especially with young children, can usually tell when hypoglycaemia occurs from the change in appearance and behaviour of the child and has to be instructed about this. Although hypoglycaemia frequently wakes the child, if it occurs during sleep at night the child may not wake and pass into coma. This is more likely to happen with young children, and the parent must always see at the parent's bedtime, that all is well.

The warning signs of an insulin reaction must never go unchecked and sugar or some form of carbohydrate taken at once.

\section{Ketosis and Coma}

Severe ketosis leading to diabetic pre-coma and coma are serious and acute diabetic emergencies. It may occur at the onset of diabetes or follow dietary indiscretions, omission of insulin or any infection. It will rapidly follow vomiting in the diabetic child from any cause. It cannot be emphasized too strongly that persistent vomiting in the diabetic child will lead to diabetic coma within a matter of hours. The child is listless, thirsty, polyuric and gravely ill. Treatment must be started immediately, preferably in hospital. Those cases in which the child is conscious and not vomiting although ketosed, are best treated by the fourhourly emergency regime. In this, glucose or lactose, which is less nauseating, is given every four hours covered by soluble insulin in dosage depending on the urine tests for sugar and ketones according to the following chart.

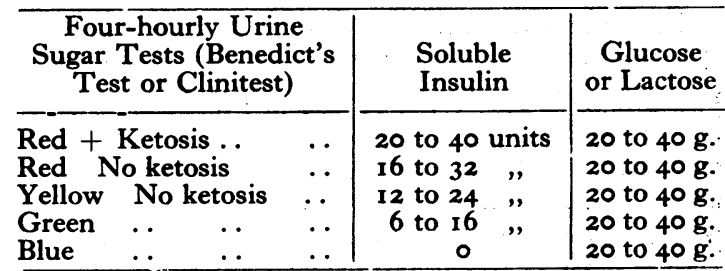

N.B.-The glucose or lactose may be given in divided amounts every two hours.

The dose of insulin depends on the age of the child and severity of symptoms. The glucose or lactose is given every four or two hours, without fail, 20 minutes after the insulin in the appropriate dose for the child.

Ketosis and diabetic symptoms should be relieved after 24 to 36 hours on this regime, after which the carbohydrates can be given at regular meal times and soluble insulin thrice or twice a day. This regime should never be continued longer than $4^{8}$ hours except for very unusual reasons.

The treatment of diabetic coma in children is much the same as for adults and has been well described elsewhere. ${ }^{2,3}$ The vital principles are to start treatment at the earliest possible moment, to give sufficient insulin part intravenously, part intra-muscularly and rapid infusion of suitable electrolyte solutions to replace water and electrolyte loss. The treatment must be controlled throughout by biochemical control with regular blood sugar estimations. Gastric lavage as soon as treatment has started may be a life-saving procedure and should never be omitted. An early enema may also help.

\section{General Management}

The greatest difficulties in managing diabetic children are not usually found in prescription of diet and insulin but in obtaining the child's willing co-operation to submit to the discipline of treatment. The parents also may be difficult. The intelligent child seldom presents any difficulty. The principles of treatment and the reasons why are easily grasped, and the improvement in health an excellent practical demonstration of the success of the treatment. The loss of good health following indiscretions in treatment further emphasize the importance of keeping to the rules. The practical experience of the improvement in health and energy after the usually unpopular change over from poor control due to the use of a long-acting insulin given once a day to the better control on two injections of soluble insulin per day is most gratifying. Few children having experienced this are willing to even consider reverting to the old treatment. 
The greatest difficulties are met with those children who will not grasp these principles and are continually eating sweets and forbidden fruits. It frequently happens that the parents of these children are also unco-operative, indifferent or stupid. Then the physician has to exercise great patience and continuous education with repeated frequent visits to the clinic until some measure of understanding is forthcoming. Great help with this difficult problem is now being given by health visitors of local authority health services in parts of London and some of the provinces: The health visitors are trained in diabetic treatment and can visit the homes and help in the day-to-day problems of diet, etc., in the home.

For those children whose health is suffering severely from either-and most commonly-lack of parental management or uncommonly from complete unco-operation of the child, there are available a number of residential hostels for diabetic children. In these hostels the child's diet and diabetic treatment is carefully supervised and the child is taught slowly and sympathetically how? to manage their own treatment.

\section{Conclusion}

The late cardio-vascular complications of diabetes are seldom seen in children. They are veryo seldom seen before the diabetes has been presentw for more than ro years. The important thing too remember in the treatment is that the child will be diabetic for the rest of its life and if this is to be $e^{\text {s }}$ long, healthy and successful, then good control of the diabetes is essential to slow down the onset and perhaps prevent in many cases, the killing complications of diabetes.

\section{REFERENCES}

I. ROBERTSON, J. (1958), "The Principles of Treatment of Diabetes in Childhood,' lecture, 3rd Congress, Internationa? Diabetes Federation, Dusseldorf.

2. NARARRO, J. D. N., SPENCER, A. G., and STOWERS J. M. (1952), Lancet, 1,983 .

3. YOUNG, B. A. 'Ketosis and Coma in Diabetes Mellitus,'

\section{Hospital Diet . . .}

However adequate, and attractive, the hospital diet, it may well be found that the patient's will to eat and power to assimilate need a little encouragement. In many cases, when it is desired to spare the body physiological effort and still provide food, Lucozade will be found valuable. It is so refreshing and stimulating to the jaded palate that it creates a new desire for solid food.
Lucozade is lightly carbonated with an attractive golden colour and a pleasant citrus flavour. It contains $23.5 \% \mathrm{w} / \mathrm{v}$ Liquid Glucose and its energy value is $2 I$ Calories per fluid ounce. It is supplied in $6 \mathrm{oz}$. and $26 \mathrm{oz}$. bottles. 